\title{
Mastite por Candida em lactante: um relato de caso
}

Carolina Boavida Ferreira, ${ }^{1}$ Sara Andrade ${ }^{2}$

\section{RESUMO}

Introdução: O leite materno é o alimento mais indicado para o bebé, em exclusividade até aos seis meses de vida, devendo ser mantido até aos dois anos de idade. No entanto, para muitas mulheres, as dificuldades no aleitamento materno resultam na sua cessação precoce. A dor mamária e a dor mamilar são queixas frequentes da lactante e dos motivos mais frequentes de interrupção da amamentação. A infeção por Candida é uma causa de dor mamária e mamilar de difícil diagnóstico. Este caso clínico aborda as características da dor mamária desta infeção fúngica, bem como o seu diagnóstico, fatores de risco e tratamento. Descrição do caso: Mulher, 36 anos, saudável, tem o primeiro filho em abril de 2016 (gravidez e parto sem complicações). A realizar amamentação exclusiva, indolor, até o bebé ter cerca de um mês, altura em que inicia dor na mama direita. Recorre a dois médicos, de especialidades diferentes, tendo sido medicada com flucloxacilina oral. Por ausência de melhoria e pelas queixas mantidas recorre ao médico de família. Refere dor intensa nos quadrantes inferiores, tipo facada, com agravamento após a mamada. O exame objetivo da mãe e do bebé são inespecíficos. Pela semiologia apresentada, gravidade do quadro e duração das queixas opta-se por prova terapêutica com fluconazol oral (lactante) e miconazol gel oral (bebé), com cuidados higiénicos associados. Após duas semanas de terapêutica, a mãe fica assintomática e a amamentar sem dor.

Comentário: O diagnóstico e o tratamento da mastite a Candida em lactantes são desafiantes pela variedade de semiologia possível. A evidência científica sobre este tema é escassa, havendo poucos estudos que confirmem laboratorialmente a presença de Candida no leite materno em lactantes sintomáticas. É importante que os médicos de família estejam atentos à semiologia desta infeção e ao sofrimento que esta poderá causar. Neste caso clínico, a prova terapêutica veio a confirmar a hipótese diagnóstica de mastite a Candida e reduzir o sofrimento materno-fetal.

Palavras-chave: Aleitamento materno; Mastite.

\section{INTRODUÇÃO}

1 interação mãe-filho é uma relação complementar, uma díade, que está em permanente mudança e que idealmente fornece à criança tudo o que ela precisa para um desenvolvimento pleno.

A mastite é uma patologia relativamente frequente na mulher que amamenta. Surge maioritariamente nas primeiras seis semanas após o parto (prevalência entre 75-95\% antes do bebé completar os três meses de vida), podendo, contudo, ocorrer ao longo de todo o período da amamentação. ${ }^{1-3}$

A sua incidência é variável nas populações. Na Austrália, onde $80 \%$ das mulheres amamenta e $50 \%$ das mulheres se encontra a amamentar aos seis meses pós-parto, estudos populacionais relatam uma incidência

1. Médica Interna de Medicina Geral e Familiar. USF São Julião.

2. Médica Interna de Medicina Geral e Familiar. USF Conde de Oeiras. de mastite de $15-20 \%$ nos primeiros seis meses. ${ }^{4}$ Já nos EUA, um estudo de coorte americano que acompanhou 946 lactantes, verificou que $9,5 \%$ destas mulheres tiveram mastite ao longo dos três meses pós-parto. ${ }^{2,5}$

A mastite a Candida é uma causa pouco frequente, sem diagnóstico preciso e mesmo contestada por alguns autores, devido à inexistência de estudos de boa qualidade ou com confirmação laboratorial microbiológica. ${ }^{6-7}$ Um estudo americano de 1996 estimou que cerca de $18 \%$, de um total de 61 lactantes com dor mamilar, tinham mastite a Candida. ${ }^{6,8}$

Este caso clínico tem como objetivo principal alertar para a dificuldade diagnóstica da mastite a Candi$d a$ e para a importância do seu tratamento.

\section{DESCRIÇÃO DO CASO}

Utente do sexo feminino, 36 anos, casada, membro de uma família nuclear, fase II do ciclo de Duvall, pro- 
fessora. Sem alergias conhecidas, consumo de álcool ou tabaco, está medicada com contracetivo oral (desogestrel $75 \mu \mathrm{g} / \mathrm{dia}$ ). Como antecedentes pessoais há a salientar apenas escoliose. G1P1 com bebé do sexo masculino, nascido em abril de 2016, parto eutócico, sem complicações, a fazer aleitamento materno exclusivo.

Vem a uma consulta de doença aguda no final de junho de 2016, por dor na mama direita com cerca de 1,5 mês de evolução (2,5 meses pós-parto). Já havia recorrido a dois médicos de diferentes especialidades: o primeiro não objetivou sinais de candidíase no bebé ou alterações mamárias na mãe que justificassem terapêutica; no segundo foi medicada com flucloxacilina oral, 500mg de 8 em 8 horas, durante sete dias, sem melhoria. Segundo a utente, as características da dor e as alterações mamárias mantiveram-se iguais, à exceção da dor, que teve um agravamento progressivo. Assim, duas semanas após o término do antibiótico recorre à Unidade de Saúde Familiar, referindo agravamento álgico, e pondera cessar a amamentação. Apresenta dor localizada nos quadrantes inferiores da mama direita, tipo facada, intensa, com agravamento após a mamada. Sem febre ou outras queixas. À observação mamária detetam-se apenas achados inespecíficos, como ligeira hiperémia e calor da pele dos quadrantes inferiores. O mamilo não tinha quaisquer alterações (antes e após a mamada). A palpação mamária/mamilar era indolor e não se palpavam nódulos ou massas. No bebé não se objetivam sinais de candidíase e a pega aparentava ser correta.

É medicada com paracetamol para as dores e recomenda-se aplicação de gelo após a mamada, informando-se de que iria ser contactada no dia seguinte, após o estudo do caso. Assim, depois da pesquisa de bibliografia adequada e respetiva integração com os dados colhidos na consulta, conclui-se que a hipótese diagnóstica de mastite ductal a fungo (sendo a mais frequente mastite a Candida albicans) ${ }^{1}$ se adequa como etiologia do quadro álgico. Contacta-se a doente e propõe-se prova terapêutica com fluconazol oral, 200mg por dia (400mg no primeiro dia), durante duas a três semanas para a mãe e miconazol (gel oral) para o bebé, 2,5ml de 12 em 12 horas, durante sete dias. Como restantes cuidados recomenda-se a mudança frequente das fraldas do bebé, a exposição das mamas ao ar, a lavagem das mãos antes e após a mamada e a esteriliza- ção de chuchas, bomba de extração de leite e biberões.

Uma semana e meia depois de iniciar o tratamento, a utente volta à consulta, já sem dor e a amamentar sem problemas. Completou duas semanas de antifúngico, com melhoria completa e remissão da sintomatologia.

\section{COMENTÁRIO}

Os benefícios do aleitamento materno são inúmeros e já conhecidos. ${ }^{6,9}$ Apesar das recomendações e da evidência dos seus benefícios, a maioria das mulheres para de amamentar antes do tempo recomendado..$^{2-3,9}$ A dor mamilar e a dor mamária são das causas mais frequentes de interrupção do aleitamento materno e, de entre as suas inúmeras etiologias, a mastite a Candida é uma causa possível e pouco frequente. ${ }^{1,6,9}$

A mama da lactante e a cavidade oral do bebé estão muitas vezes colonizadas com diversas espécies de Candida, mais frequentemente a Candida albicans. ${ }^{1,6}$ Podendo ser comensal não há sintomas, mas um sobrecrescimento deste fungo, mais provável num ambiente húmido e rico em açúcar como o da mama da lactante, pode propiciar uma infeção. ${ }^{6}$ Quando esta ocorre pode ser superficial/cutânea ou profunda/ductal e afetar uma ou as duas mamas, tornando o tratamento desafiante. ${ }^{1,6}$

Na literatura estudada há referência a alguns fatores de risco, quer da mãe quer do bebé. Salienta-se, no entanto, que há poucos estudos sobre este tema, sendo que a identificação de alguns destes fatores se baseia apenas em relatos de pacientes. ${ }^{3}$ No que diz respeito à mãe, existe associação com antibioterapia recente, imunodepressão, infeções fúngicas recorrentes, ingurgitamento mamário, traumatismo mamilar, uso de mamilos de silicone ou de bombas de extração de leite, ingestão de grandes quantidades de produtos lácteos, bebidas alcoólicas, produtos açucarados ou adoçantes, entre outros. ${ }^{1-2,6,10,12}$ Como fatores de risco do bebé está descrita a existência de candidíase oral, perineal ou outra, antibioterapia recente, prematuridade, imunodepressão, utilização de chuchas ou tetinas..$^{1-2,6,10,12}$

A semiologia é muito diversa. ${ }^{1-2} \mathrm{Na}$ candidíase intraductal pode ocorrer dor mamária intensa tipo «facada», «queimadura» ou "picada de alfinete», habitualmente durante ou após a mamada, sendo o início da mamada geralmente indolor., ${ }^{6,-10,12}$ Alterações cutâneas no ma- 
milo e/ou na aréola (pele brilhante, escamosa, eritematosa, pruriginosa, edemaciada, gretada ou com placas brancas) podem estar presentes (candidíase cutânea da mama), assim como sinais de infeção fúngica no bebé (orais ou perineais), recusa alimentar, choro ou sinais de candidíase vaginal na mãe. ${ }^{1,9-10,12}$ Pode haver ausência de sintomas. ${ }^{1,6,12} \mathrm{O}$ companheiro também pode apresentar um sobrecrescimento fúngico a nível do pénis (balanite por Candida), sintomático ou não. ${ }^{1-2}$

O diagnóstico da mastite a Candida na lactante é difícil, pois é frequentemente baseado em sinais e sintomas subjetivos e inespecíficos. ${ }^{1-2,9,12}$

A cultura do leite materno é, na maioria das vezes, pouco útil, devido à dificuldade do crescimento do microrganismo no leite (é inibido pela lactoferrina). ${ }^{1-3} \mathrm{~A}$ adição de ferro ao leite evita este fenómeno, mas é um método cultural que não está habitualmente disponível na prática clínica. ${ }^{1-2}$

A evidência científica sobre o tema é escassa, são poucos os estudos que confirmaram laboratorialmente a presença de Candida. Francis-Morrill e colaboradores avaliaram a sensibilidade, a especificidade, o valor preditivo positivo (VPP) e o índice de verosimilhança dos sinais e sintomas de mastite a Candida através da confirmação da presença deste fungo no mamilo, aréola ou no leite de 100 lactantes. Concluíram que o VPP era maior na presença de três ou mais sinais ou sintomas em simultâneo, ou se coexistirem alterações cutâneas do mamilo e da aréola, ou na presença de uma destas alterações associadas a dor mamária. No entanto, um VPP superior a $70 \%$ e a decisão de tratamento antifúngico baseada só em sinais e sintomas não considera a possibilidade de etiologias mistas (bacteriana e fúngica) ou de resistência ao tratamento escolhido., ${ }^{2,7}$

Assim, a suspeita de mastite a Candida deve ser considerada quando a dor tem as características acima descritas e é desproporcional aos achados físicos, estando frequentemente associada a infeções fúngicas do bebé ou da mãe. ${ }^{3,-10}$

A observação da mamada é também essencial, uma vez que grande parte das dores mamárias da lactante são devidas a uma má pega, facilmente corrigível e o esvaziamento completo da mama reduz o risco de mastite. $^{3,9,11}$

Outras hipóteses diagnósticas, como vasoconstrição mamilar (pode ocorrer em lactantes que têm fenóme- no de Raynaud ou aumento da sensibilidade mamilar), ingurgitamento mamário, abcesso mamário, galactocelo ou traumatismo mamilar, devem ser colocadas e excluídas através da história clínica e exame objetivo. ${ }^{1-2,9,11}$ A hipótese de mastite bacteriana a Staphylococcus aureus, particularmente às estirpes resistentes à meticilina (MRSA), também deve ser descartada, denotando-se que nesta patologia há geralmente associação a mialgias, fadiga, cefaleia e febre $\left(38,5^{\circ} \mathrm{C}\right) .^{3,12-13} \mathrm{Têm}$ sido realizados diversos estudos no sentido de perceber melhor quais os microrganismos responsáveis por esta mastalgia. Surpreendentemente, em grande parte dos casos são causadas por estafilococos coagulase-negativos e estreptococos, muitos destes comensais da mama materna e/ou flora oral do bebé. ${ }^{13}$ Não produtores de toxinas (responsáveis por reação inflamatória local ou sistémica), a semiologia nestes casos é ligeira, podendo ocorrer dor tipo queimadura e sensação de produção de pouco leite, não ocorrendo hiperémia mamária. ${ }^{13} \mathrm{O}$ carcinoma inflamatório da mama deve ser considerado quando coexiste linfadenopatia axilar e no caso de o tratamento adequado da eventual mastite não resolver o quadro. ${ }^{3,9}$

No que diz respeito à abordagem terapêutica da mastite a Candida, a mãe e o bebé devem ser tratados simultaneamente, mesmo que o bebé não apresente sinais de candidíase. ${ }^{2-3}$ Se o companheiro tiver queixas, também deve ser medicado. O esquema terapêutico proposto é baseado na experiência clínica e na opinião de peritos em aleitamento materno..$^{9-10,12}$

Inicialmente o tratamento consiste em:

- Bebé: miconazol gel oral (posologia dependente da idade) ou nistatina em suspensão oral (100.000 UI, quatro vezes por dia, após as mamadas), durante duas semanas ou até ausência de sintomas há uma semana. ${ }^{2-3,6,9,12}$ É preferível evitar o uso da nistatina devido ao número elevado de resistências (40\%). ${ }^{2,6,10}$

- Mãe: miconazol, clotrimazol ou cetaconazol tópicos, durante duas semanas, com massagem suave mamilar após cada mamada (e remoção antes da mamada seguinte, no caso de clotrimazol e cetoconazol)., ${ }^{2,6,9-10,12}$

O violeta de genciana a $1 \%$ (solução em água) também pode ser utilizado como agente inicial se a dor for intensa ou quando não há resposta aos agentes tópicos acima referidos. Apesar de ser uma opção bastante eco- 
nómica é pouco usado, pois mancha muito. Este deve ser aplicado nos mamilos, aréolas e na boca da criança com um cotonete, uma vez por dia, durante três a quatro dias. ${ }^{3,9,11-12}$

Se a dor persistir, as alternativas são:

- Bebé: terapêutica tópica (igual ao tratamento inicial) ou fluconazol oral (posologia dependente do peso da criança). ${ }^{2-3,6,9,12}$

- Mãe: fluconazol oral (400mg no primeiro dia), seguida de 200mg por dia ou nistatina em suspensão oral (500.000 UI quatro vezes por dia), durante duas semanas ou até ausência de sintomas há uma semana. ${ }^{2-3,6,9-12}$

Está recomendada a manutenção do aleitamento materno durante o tratamento. ${ }^{2}$ Regista-se alguma relutância na prescrição de fluconazol oral a lactantes, sendo, no entanto, este fármaco seguro durante a amamentação. ${ }^{2,6,9}$

É essencial expor as mamas ao ar, lavar as mãos antes e após a muda de fralda e a amamentação, esterilizar chuchas, mamilos de silicone, bombas mamárias ou biberões. ${ }^{1,6,10}$

Todos os médicos que tratam lactantes devem estar atentos à possibilidade de mastite a Candida como causa de dor mamária. ${ }^{6}$ Esta patologia, apesar de pouco frequente, é responsável por grande sofrimento da díade mãe-filho e, muitas vezes, pela cessação do aleitamento materno. ${ }^{6}$

Existe pouca evidência científica no que diz respeito a fatores de risco, diagnóstico e tratamento desta infeção fúngica.

Este caso clínico aborda um subtipo de mastite, patologia frequente embora de diagnóstico nem sempre fácil. De entre os inúmeros diagnósticos possíveis, a suspeição diagnóstica de mastite intraductal a Candi$d a$ foi mais forte, devido à história clínica, às alterações ao exame objetivo e à evolução clínica do quadro apresentado pela lactante: dor intensa com mês e meio de evolução, a agravar; a terapêutica antibiótica já efetuada (flucloxacilina, antibiótico correto para Streptococcus e Staphylococcus não meticilino-resistentes) não surtiu qualquer efeito; não havia febre ou sinais de vasospasmo; durante bastante tempo a utente esteve sem medicação e, portanto, a terapêutica conservadora não resolveria a situação; as queixas eram compatíveis com mastite ductal a Candida, apesar de toda a incerteza deste diagnóstico. Assim, com a iminência de parar de amamentar, o elevado sofrimento apresentado e a clínica compatível, optou-se por realizar uma prova terapêutica com fluconazol oral. Idealmente, a mãe deveria ter sido informada da falta de evidência desta patologia e dos eventuais riscos do seu tratamento sem a confirmação laboratorial. Em lactantes que fazem outros medicamentos pode ser importante a marcação de uma consulta prévia à prescrição de fluconazol, de modo a inferir sobre eventuais interações medicamentosas. Se a terapêutica com este antifúngico for prolongada poderá ser necessária uma avaliação dos valores das enzimas hepáticas e, havendo um aumento, uma monitorização mais apertada deverá ser realizada para prevenir a toxicidade hepática (sendo a maioria reversível com a suspensão do fármaco).$^{14}$ Os benefícios do tratamento devem também ser ponderados. Sendo saudável a lactante deste caso clínico, a suspeita de mastite a Candida muito forte e o tratamento não prolongado, considerou-se que os benefícios foram superiores aos riscos. O tratamento efetuado veio a revelar-se eficaz na supressão das queixas e do sofrimento da lactante.

Pela continuidade de cuidados e proximidade com o utente, o médico de família encontra-se numa posição privilegiada para abordar problemas do puerpério e da maternidade e acompanhar de perto os riscos/benefícios da sua atitude, não esquecendo que no centro da sua decisão está sempre o utente.

\section{REFERÊNCIAS BIBLIOGRÁFICAS}

1. Zeretzke K. Yeast infections and the breastfeeding family: helping mothers find relief for symptoms and treatment for the infection preserves the breastfeeding relationship. Leaven. 1998;34(5):91-6.

2. Wiener S. Diagnosis and management of Candida of the nipple and breast. J Midwifery Womens Health. 2006;51(2):125-8.

3. Spencer JP. Management of mastitis in breastfeeding women. Am Fam Physician. 2008;78(6):727-31.

4. Amir LH, Forster DA, Lumley J, McLachlan H. A descriptive study of mastitis in Australian breastfeeding women: incidence and determinants. BMC Public Health. 2007;7:62.

5. Foxman B, D'Arcy H, Gillespie B, Bobo JK, Schwartz K. Lactation mastitis: occurrence and medical management among 946 breastfeeding women in the United States. Am J Epidemiol. 2002;155(2):103-14.

6. Hanna L, Cruz SA. Candida mastitis: a case report. Perm J. 2011;15(1): 62-4.

7. Francis-Morrill J, Heinig MJ, Pappagianis D, Dewey KG. Diagnostic value of signs and symptoms of mammary candidosis among lactating women. J Hum Lact. 2004;20(3):288-95. 
8. Amir LH, Garland SM, Dennerstein L, Farish SJ. Candida albicans: is it associated with nipple pain in lactating women? Gynecol Obstet Invest. 1996;41(1):30-4.

9. Spencer J. Common problems of breastfeeding and weaning [Internet]. UpToDate; updated 2018 Jul 17. Available from: https://www. uptodate.com/contents/common-problems-of-breastfeeding-andweaning

10. La Leche League International. Is thrush causing my sore nipples? [homepage]. Raleigh: La Leche League International; 2019. Available from: https://www.lli.org/breastfeeding-info/thrush/

11. Newman J. Candida protocol [homepage]. Toronto: International Breastfeeding Center; 2009 [updated 2017 Jun]. Available from: https://ibconline.ca/information-sheets/candida-protocol/

12. Gonzalez C. Manual prático de aleitamento materno. Rio de Janeiro: Timo; 2014. ISBN 9788569404019

13. Jiménez E, Arroyo R, Cárdenas N, Marín M, Serrano P, Fernández L, et al. Mammary candidiasis: a medical condition without scientific evidence? PLoS One. 2017;12(7):e0181071.

14. Agência Europeia do Medicamento. Fluconazol: resumo das carac- terísticas do medicamento [Internet]. Londres: EMA. Available from: http://www.ema.europa.eu/docs/pt_PT/document_library/Referrals_document/Fluconazol_Tiefenbacher_29/WC500010808.pdf

\section{CONFLITO DE INTERESSES}

As autoras declaram não ter quaisquer conflitos de interesse.

\section{FINANCIAMENTO}

O trabalho relatado neste manuscrito não foi objeto de qualquer tipo de financiamento externo (incluindo bolsas de investigação).

\section{ENDEREÇO PARA CORRESPONDÊNCIA}

Carolina Boavida Ferreira

E-mail: carolinagbf@gmail.com

http://orcid.org/0000-0002-6392-7466

Recebido em 30-11-2016

Aceite para publicação em 25-07-2018

\section{ABSTRACT}

\section{CANDIDA MASTITIS IN A BREASTFEEDING WOMAN: A CASE REPORT}

Introduction: Breast milk is the most suitable food for the baby, exclusively until six months of age, and should be kept up to two years of age. However, for many women, difficulties in breastfeeding result in its premature discontinuation. Breast and nipple pain are frequent complaints and the most frequent reasons for discontinuation of breastfeeding. Candida infection is a cause of breast and nipple pain as represents a challenging diagnosis. This case report addresses the characteristics of breast pain in the context of this fungal infection, as well as its diagnosis, risk factors, and treatment.

Case report: Female, 36 years old, healthy, had her first child in April 2016 (uncomplicated pregnancy and childbirth). Exclusive breastfeeding, without pain, until the baby was about a month old, when she started began complaining breast pain (right breast). She was observed by two different specialists and was medicated with oral flucloxacilin. Due to the lack of improvement and the ongoing complaints, she decides to go to her primary care center. The pain was very intense, like stabbing, and worsened after feeding her baby. The physical examination of both mother and baby was non-specific.

Due to the semiology presented, severity of the symptoms and duration of complaints, oral fluconazole was prescribe to the mother, and oral miconazole gel for the baby, with as well as usual personal hygiene care. After two weeks of treatment, the woman became asymptomatic and was able to breastfeed without pain.

Comment: The diagnosis and treatment of Candida mastitis in breastfeeding women are challenging due to the variety of symptoms that women may experience. The scientific evidence is scarce, with few studies confirming the presence of Candida in the breast milk. It is important for family doctors to be aware of this infection and the suffering it may cause. In this clinical case, the results of the therapeutic choice confirmed the diagnostic hypothesis of Candida mastitis and reduced maternal and fetal distress.

Keywords: Breastfeeding; Mastitis. 